\title{
EpCAM Antibody-conjugated Mesoporous Silica Nanoparticles to enhance the Anticancer Efficacy of Carboplatin in Retinoblastoma
}

Wei $\mathrm{Qu}^{1}$, Bo Meng ${ }^{1}$, Yangyang $\mathrm{Yu}^{2}$, Shaowei Wang ${ }^{1 *}$

${ }^{1}$ Department of Ophthalmology, The $2^{\text {nd }}$ Affiliated Hospital of Haerbin Medical University, Haerbin, Heilongjiang 150086, China

${ }^{2}$ Department of Ophthalmology, Heilongjiang Provincal Hospital, Haerbin, Heilongjiang 150086, China

\section{*Corresponding author:}

Shaowei Wang,

Department of Ophthalmology,

The $2^{\text {nd }}$ Affiliated Hospital of Haerbin Medical University, Haerbin, Heilongjiang 15008, China Tel/Fax: +86 45186662961

Email: shaoweiwang123@ hotmail.com 


\begin{abstract}
Retinoblastoma $(\mathrm{RB})$ is a rare type of tumor which occurs in the intraocular spaces of eye. Carboplatin (CRB) has been used for the treatment of RB along with focal therapy. In this study, we have demonstrated the preparation of EpCAM-conjugated mesoporous silica nanoparticles for the successful delivery of carboplatin (CRB) to the retinoblastoma cells. The particles size and morphology was nanosized and spherical and exhibited a controlled release kinetics. Cellular uptake studies reveal that enhanced internalization was observed for EpCMSN compared to that of CMSN. The remarkable difference in cellular uptake might be attributed to the specific receptor mediated cellular internalization for EpCMSN compared to that of CMSN which might entered the cells by passive diffusion. Consistently, EpCMSN exhibited a superior anticancer effect in retinoblastoma cells. The IC50 value of EpCMSN was $1.38 \mu \mathrm{g} / \mathrm{ml}$ which is significantly lesser compared to that of free CRB $(3.26 \mu \mathrm{g} / \mathrm{ml})$. EpCMSN resulted in 2-fold increase in caspase-3 level compared to that of free CRB. The higher targeting effect of EpCAM receptor resulted in enhanced apoptosis of cancer cells after treatment with EpCMSN in retinoblastoma cells. Taken together, EpCAM conjugated MSN might be advantageous for the targeted treatment of receptor-overexpressed ocular malignancies like retinoblastoma.
\end{abstract}

\title{
Keywords
}

Retinoblastoma, carboplatin, mesoporous silica nanoparticles, anticancer effect 


\section{Introduction}

Retinoblastoma (RB) is a rare type of tumor which occurs in the intraocular spaces of eye [1]. The incidence rate of RB is so rare such that only 1 in 20,000 births will have this malignancy. The RB cases were mainly observed in developing countries like China compared to that of welldeveloped countries [2,3]. The poor survival rate of RB was mainly due to the delayed diagnosis of the rare tumor and inadequate healthcare system. Generally RB is associated with the mutation of retinoblastoma gene (RB1) in approximately $40 \%$ of patients. Although RB could be treated however risk of secondary tumor which could occur many years after the first tumor is a serious challenge [4]. Although initially, enucleation was the main treatment strategy however later on radiotherapy and focal therapy has been evolved. It has been reported that such additional therapies often resulted in severe side effects such as deformations, cataract and retinopathy $[5,6]$. Chemotherapy if appropriately used could be a conservative treatment strategy and could reduce the tumor size [7].

Recently, carboplatin has been used for the treatment of RB along with local therapy $[8,9]$. CRB exhibits the anticancer effect by crosslinking the DNA which in turn results in the cellular apoptosis as the repair become unsuccessful [10]. As with all anticancer drugs, CRB is not free from side effects and results in severe side effects including nephrological and neurological toxicities, neutropenia, and thrombocytopenia [11,12]. In this context, efforts have to be made to deliver the anticancer drugs right in the area of interest and to avoid unnecessary side effects. Drug delivery in the eye is a big challenge for the clinicians for the numerous reasons as it is well-protected from various physiological barriers. Among all the available strategy, delivery of drug using a nanomedicine approach would be ideal [13]. The nanoparticles are small nanosized particles made up of polymer or lipid or inorganic materials that can encapsulate the anticancer 
drugs and release the loaded content in a sustained manner. To be specific, biodegradable nanoparticles are gained increasing interest owing to the need to be compatible with all the organs and tissues (especially eye) [14]. Among all the carriers, mesoporous silica nanoparticles (MSN) attracted the attention of researchers owing to its excellent properties including biocompatibility, surface modifications, high chemical stability, unique mesopores and high surface area [15]. The drug loaded in the mesopores of MSN could be released in the desired region over a prolonged period of time [16]. As mentioned above, treatment in the eye presents several serious challenges; therefore, we have designed the carrier with high specificity towards RB. Epithelial cell adhesion molecule (EpCAM), a transmembrane protein is reported to overexpress in the $\mathrm{RB}$ of the eye and there are reports suggesting that the inhibition of EpCAM results in poor cell proliferation $[17,18]$. Therefore, EpCAM could be an ideal target to increase the therapeutic efficacy against RB cancers. EpCAM itself consists of two EGF-like extracellular domains [19]. Therefore, targeting of EpCAM receptors offers numerous advantages including (a) it is overexpressed in epithelial cancers (b) apical expression in tumor cells and (c) increased target specificity [20].

We hypothesized that EpCAM surface modified MSN would increase the anticancer efficacy of $\mathrm{CRB}$ in retinoblastoma cancers while at the same time various side effect of CRB would be significantly minimized. For this purpose, CRB was loaded in the MSN which contains carboxylic acid $(\mathrm{COOH})$ group on the outer surface which was then used to conjugate amine group of EpCAM. The particle size, shape, and release parameters were studied in detail. The target specificity of MSN was studied by cellular uptake analysis and efficacy was studied by means of cytotoxicity assay and apoptosis analysis and caspase- 3 analysis. 


\section{Materials and Methods}

\section{Materials}

N-Cetyltrimethylammonium bromide (CTAB), Tetraethylorthosilicate (TEOS, 28\%), sodium hydroxide $(\mathrm{NaOH})$, and 3-(Trimethoxysilyl)propyl methacrylate (MPS, 99\%) was purchased from Sigma-Aldrich, China. EpCAM antibody was purchased from Santa Cruz, CA. Carboplatin was also purchased from Sigma-Aldrich, China. All other chemicals are of reagent grade.

\section{Preparation of EpCAM-conjugated mesoporous silica nanoparticles}

First, functional group modified MSN was prepared by sol-gel method using TEOS as silica precursor, $\mathrm{CTAB}$ as a surfactant, and $\mathrm{NaOH}$ as a base or catalyst. Briefly, $2.74 \mathrm{mmol}$ of $\mathrm{CTAB}$ was dissolved in $480 \mathrm{ml}$ of distilled water and $3.6 \mathrm{ml}$ of $\mathrm{NaOH}(2 \mathrm{M})$ was added and stirred until a clear solution as observed. The aqueous suspension of carboplatin was dissolved and the solution temperature was adjusted to $80^{\circ} \mathrm{C}$ and followed by that TEOS $(5 \mathrm{ml})$ was added in a dropwise manner. The solution was vigorously stirred for $2 \mathrm{~h}$ until a white precipitate was formed. The product was collected by centrifugation at $15000 \mathrm{rpm}$ for $10 \mathrm{~min}$. The particles were washed in water and ethanol for several cycles. The final product was directly added to the ethanol solution containing $0.5 \mathrm{ml}$ of MPS. The solution was stirred for $12 \mathrm{~h}$ at room temperature. Followed by, synthesized product was stirred in ethanol and the final product was collected. The EpCAM antibody was conjugated on the surface of MSN via an EDC/NHS reaction. Briefly, 50 mg of COOH-modified MSN was dissolved in $5 \mathrm{ml}$ of PBS and dissolved by stirring. EDC and NHS in equimolar concentration was added and left to the room temperature for $6 \mathrm{~h}$. The sample

was centrifuged to remove the excess EDC and NHS and washed with PBS two times. $500 \mu 1$ of EpCAM- antibody (200 $\mu \mathrm{g} / \mathrm{ml}$ in PBS) was added to the NP dispersion and allowed to react for 
6h. The so-formed product was centrifuged to remove the unconjugated antibody and the final product was stored.

\section{Particle size analysis}

The particle size and polydispersity index of the nanoparticle was evaluated by Malvern Instruments, Malvern, UK (Zeta Sizer) using a manufacturer's software (NanoDTS, version 6.34) employing a cumulant analysis method. The data are presented as mean \pm standard deviation.

\section{Morphology analysis}

The morphology of nanoparticle was studied by transmission electron microscope (TEM). The sample was loaded onto a copper grid and then counterstained with $2 \%$ phosphotungistic acid (PTA). The sample was dried and then observed under TEM (H-7600, Hitachi, Japan).

\section{In vitro drug release study}

The drug release study was performed in phosphate buffered saline $(\mathrm{pH} 7.4)$ at $37^{\circ} \mathrm{C} .10 \mathrm{mg}$ of drug-loaded sample was dispersed in PBS (1 $\mathrm{ml})$ and packed in a dialysis tube. The dialysis tubes were placed in a shaker maintained at $37^{\circ} \mathrm{C}$ and $100 \mathrm{rpm}$. The samples were collected at specified time interval. The dialysis tubes were centrifuged at high speed for $10 \mathrm{~min}$ and the supernatant was collected to estimate the amount of drug released in the media. The pellet was again dispersed in equal amount of media and put back in the shaker bath. The amount of drug released at specified time was estimated by HPLC method. A high-performance liquid chromatography (HPLC; Hitachi, Japan) was used for CRB analysis; a reverse-phase Hypersil Gold C8 column (GL science, $5 \mu \mathrm{m}, 4.6 \mathrm{~mm} \times 250 \mathrm{~mm}$ ) and a UV vis detector (L-2420) were 
used. A mobile phase consisting of acetonitrile and water (50:50) was used and a flow rate of 1 $\mathrm{ml} / \mathrm{min}$ was used.

\section{Cell culture}

The human retinoblastoma cell (Y79) was purchased from the cell bank, China. The cancer cell was grown in RPMI 1640 growth media supplemented with $10 \%$ fetal bovine serum and $1 \%$ antibiotic mixture.

\section{Cellular uptake analysis}

To perform cellular uptake assay, Y79 cancer cells were seeded in a 12-well plate and incubated overnight. The cells were then incubated with CMSN and EpCMSN as non-targeted and targeted nanoparticles. Rhodamine-B was used as a fluorescent probe and loaded in the MSN carrier. The cancer cells were incubated with respective formulations for $2 \mathrm{~h}$ and the cells were washed two times. The cells were extracted using trypsin and cell pellets were collected. The cell pellet was resuspended in phosphate buffer saline (pH 7.4) and analyzed using flow cytometer (FACS) (BD Bioscience Mountain View, CA, USA).

The qualitative cellular uptake (subcellular distribution) was determined by confocal laser scanning microscopy (CLSM). Y79 cancer cells were seeded in a 6-well plate and incubated overnight. The cells were then incubated with EpCMSN for $2 \mathrm{~h}$. The cells were washed 3 times and fixed with $4 \%$ paraformaldehyde (PFA). The cells were mounted on a glass slide and the

amount of cellular uptake as observed using confocal microscope (Meta LSM510 device (Carl Zeiss, Jena, Germany)). 


\section{Cytotoxicity assay}

The cytotoxic potential of individual formulation was determined by MTT (3-(4,5dimethylthiazol-2-yl)-2,5-diphenyl tetrazolium bromide) assay. The assay is based on the principle of cleavage of tetrazolium salt to formazan salt for which absorbance was measured. Y79 cells at a seeding density of $1 \times 10^{4}$ cells/well was seeded in 96-well plate and incubated for 24h. These cells were treated with free CRB, CMSN and EpCMSN and incubated for additional 24h. Next day, cells were washed and treated with MTT solution $(20 \mu \mathrm{l}, 5 \mathrm{mg} / \mathrm{ml})$ and incubated for 4h. The cells were then added with DMSO and kept aside for $15 \mathrm{~min}$. The absorbance of each well was measured using ELISA reader at a wavelength of $570 \mathrm{~nm}$. The IC50 value of each formulation was calculated using SPSS software.

\section{Apoptosis assay by flow cytometer}

The cellular apoptosis was determined by Annexin V FITC kit (BD Biosciences). Briefly, Y79 cells at a seeding density of $2 \times 10^{5}$ cells/well was seeded in 12 -well plate and incubated for $24 \mathrm{~h}$. These cells were treated with free CRB, CMSN and EpCMSN and incubated for additional 24h. The cells were then washed and extracted using trypsinization. The cells were washed again and centrifuged. The cell pellets were re-dispersed in $100 \mu \mathrm{l}$ of binding buffer solution and added with Annexin V FITC kit as per the manufacturer's standard protocol. The cell pellets redispersed in $1 \mathrm{ml}$ of phosphate buffered saline and observed through flow cytometer (FACS) (BD Bioscience Mountain View, CA, USA).

\section{Caspase-3 analysis}

$5 \times 10^{5}$ cells were seeded in the each well of 6 -well plate and incubated overnight. These cells were treated with free CRB, CMSN and EpCMSN and incubated for additional 24h. The cells 
were extracted and washed thrice with PBS buffer. The cells were lysed using an lysis buffer ((20 mM Tris- $\mathrm{HCl} \mathrm{pH}$ 7.4, $0.1 \mathrm{mM}-$ Mercaptoethanol, $1.0 \mathrm{mM} \mathrm{MgCl} 2,0.1 \mathrm{mM}$ EDTA, 5\% glycerol, $0.1 \%$ Triton X-100, $0.5 \mathrm{mM} \mathrm{KCl}, 1 \mathrm{mg} \mathrm{ml}-1$ pepstatin and leupeptin, $0.5 \mathrm{mM}$ PMSF)) and proteins were collected. The amount of proteins was estimated using BCA protein assay kit. $100 \mu \mathrm{g}$ of protein was treated with caspase buffer containing caspase-III substrate $(5 \mu \mathrm{g})$ and incubated for $1 \mathrm{~h}$ at $37^{\circ} \mathrm{C}$. The whole mixture was then estimated for the fluorescence experiments.

\section{Statistical analysis}

An independent sample $t$-test was performed to determine the significance of differences between different groups. Differences were considered statistically significant at $P<0.05$.

\section{Results and Discussion}

In this study, we have designed the carrier with high specificity towards RB. We hypothesized that EpCAM surface modified MSN would increase the anticancer efficacy of CRB in retinoblastoma cancers while at the same time various side effect of CRB would be significantly minimized (Figure 1).

\section{Physicochemical analysis}

The drug-loaded MSN was prepared by sol-gel method using TEOS as silica precursor, CTAB as a surfactant, and $\mathrm{NaOH}$ as a base or catalyst. Functionalization of targeting ligand on the surface of MSN would enhance the internalization of nanocarrier system. Conjugation of EpCAM on the MSN surface increase the tumor cell uptake in EpCAM receptor overexpressed tumor cells. The 
EpCAM was covalently conjugated to the MSN surface using the interaction of amine and carboxylic group in the presence of EDC/NHS chemistry. The average particle size of EpCMSN was $148.5 \pm 2.35 \mathrm{~nm}$ with a uniform dispersion index of 0.15 (PDI) (Figure 2a). The particle size slightly increased from $\sim 120 \mathrm{~nm}$ to $\sim 150 \mathrm{~nm}$ for the EpCMSN indicating the definite conjugation of targeting ligand. Nevertheless, a size less than $150 \mathrm{~nm}$ would be suitable for cancer targeting applications and could potentially avoid the opsonization process in the systemic circulation. The zeta potential of EpCMSN was observed to be around $-20.1 \pm 2.15 \mathrm{mV}$. The particle shape was confirmed by TEM imaging. As revealed by TEM, particles were spherical in nature and unfirmly distributed in the TEM grid (Figure 2b). All particles were perfectly spherical indicating the success of formulation technique. The smooth and spherical nature of the particles would be beneficial for the cancer targeting applications. The conjugation of EpCAM with MSN was confirmed by gel electrophoresis. As seen (Figure 2c), free antibody was present at the bottom while $100 \%$ conjugation of antibody resulted in no movement of the band indicating the successful conjugation process. We have used the same nanoparticles for rest of the physical and biological studies.

\section{In vitro drug release}

An effective drug delivery system is the one which does not react to encapsulated drug while at the same time protects it from premature release (Figure 3). Sustained release of encapsulated compound will enable the better therapeutic efficacy in the respective tumors. The in vitro release of CRB from EpCMSN was estimated by dialysis method. As seen, a controlled release profile of CRB was throughout the study period. Approximately $\sim 30 \%$ of encapsulated drug was released within $24 \mathrm{~h}$ while around $\sim 80 \%$ of drug released by the end of $100 \mathrm{~h}$. There is no burst release pattern indicating that the entire drug encapsulated in the nanoparticle system. Such a 
sustained released of drug will be beneficial for the cancer treatment while it will reduce the associated side effects in the blood stream.

\section{Cellular uptake analysis}

In order to evaluate the targeting efficiency of EpCMSN and CMSN, we have performed cellular uptake analysis in retinoblastoma cells. Rhodamine-B was loaded in the MSN carrier as a fluorescent probe. Results clearly reveal that enhanced cellular uptake was observed for EpCMSN compared to that of CMSN (Figure 4). The remarkable difference in cellular uptake might be attributed to the specific receptor mediated cellular internalization for EpCMSN compared to that of CMSN which might entered the cells by passive diffusion. An enhanced cellular uptake of NP indicates that the increased concentration of CRB would be accumulated in the cancer cells and might produce higher therapeutic effect $[21,22]$.

The cellular uptake (subcellular distribution) was further studied by confocal laser scanning microscopy (CLSM) (Figure 5). Lysotracker Green was used to track the cellular uptake of NP in the lysosomal region. The results clearly reveal that the red fluorescence of NP exactly merged with the green fluorescence from Lysotracker Green indicating the presence of the delivery system in the lysosome. Results further indicate the NP was internalized via receptor-mediated cellular uptake. The lysosomal localization of NP ascertains that it follows the endocytosis route by which drug will be released in a controlled manner.

\section{Cytotoxicity assay}

The cytotoxicity assay of different formulations was evaluated by MTT assay. As shown (Figure 6), all formulations exhibited a typical antiproliferative effect on a dose-dependent basis. The CMSN showed higher anticancer effect than that of free CRB while EpCMSN exhibited a 
superior anticancer effect in retinoblastoma cells. The IC50 value of EpCMSN was $1.38 \mu \mathrm{g} / \mathrm{ml}$ which is significantly smaller compared to that of free CRB $(3.26 \mu \mathrm{g} / \mathrm{ml})$. The higher cytotoxicity of EpCMSN is in correlation with the cellular uptake pattern in which ligand targeted NP showed a significantly higher cellular internalization. Therefore it is clearly revealed that higher anticancer effect o EpCMSN was due to the encapsulation of drug in the nanocarrier and further conjugation of targeting moiety on the surface. The result is very important from the therapeutic efficacy perspective as CRB is considered to be an important chemotherapeutic drug in RB treatment and as such treatment resulted in many side effects. Therefore, present study was an attempt to deliver the drug in a nanocarrier which is targeted to the RB. It can be expected that the free CRB immediately diffuse into the cancer cells while NP will enter via a more specific endocytosis pathway. The nanoparticle encapsulation of anticancer drug resulted in a higher anticancer effect owing to its ability to release the drug in a controlled manner [23,24].

\section{Caspase-3 assay}

CRB is an apoptosis inducing agent and caspase-3 will be a good indicator of the apoptosis. Generally it is known that caspase-3 is the final executioner of the entire apoptotic pathways as the enzymatic activity increase with the apoptosis which results in direct increase in caspase-3 level. As shown (Figure 7), free CRB increased the caspase-3 level compared to that of control. In the similar concentration, EpCMSN resulted in 2-fold increase in caspase-3 level compared to that of control. The increased caspase- 3 is an indicative of the increased apoptosis of retinoblastoma cells which was in turn due to the enhanced cellular internalization of EpCMSN. The caspase-3 assay results were well-corroborated with the MTT assay results.

\section{Apoptosis assay (Flow cytometer)}


The cytotoxic potential of individual formulations was further confirmed by apoptosis assay (Annexin V/PI staining protocol). The formulation-treated cells were stained with Annexin V and PI staining agent and analyzed by flow cytometer. Results clearly reveal that all formulations have definite role in inducing the apoptosis of cancer cells. The free CRB induced approximately $\sim 15 \%$ of apoptosis of cancer cells while nanoparticulate encapsulation of CRB (CMSN) induced nearly two fold higher apoptosis ( 40\%) (Figure 8$)$.

As expected, $\sim 65 \%$ of apoptosis cell was observed for the treatment of EpCMSN. Superior anticancer effect of EpCMSN might be due to the targeting effect of EpCAM on the respective receptor which is overexpressed in the Y79 cancer cells. Results clearly reveal that free drugs might be quickly eliminated from the cancer cells whereas nanoparticle-based drugs might release in a slow and sustained manner for longer time period. To be specific, targeted nanocarrier internalized better than that of non-targeted nanoparticle and therefore resulted in higher anticancer effect [25]. This kind of delivery strategy is important for the cancer treatment as the drug accumulates in the cancer cells for long time period and kills the most proportion of cancer cells.

\section{Conclusion}

In conclusion, we have demonstrated the preparation of EpCAM-conjugated mesoporous silica nanoparticles for the successful delivery of carboplatin (CRB) to the retinoblastoma cells. The particles size and morphology was nanosized and spherical and exhibited a controlled release kinetics. Cellular uptake studies reveal that enhanced internalization was observed for EpCMSN compared to that of CMSN. The remarkable difference in cellular uptake might be attributed to the specific receptor mediated cellular internalization for EpCMSN compared to that of CMSN 
which might entered the cells by passive diffusion. Consistently, EpCMSN exhibited a superior anticancer effect in retinoblastoma cells. The IC50 value of EpCMSN was $1.38 \mu \mathrm{g} / \mathrm{ml}$ which is significantly lesser compared to that of free CRB $(3.26 \mu \mathrm{g} / \mathrm{ml})$. EpCMSN resulted in 2-fold increase in caspase-3 level compared to that of free CRB. The higher targeting effect of EpCAM receptor resulted in enhanced apoptosis of cancer cells after treatment with EpCMSN in retinoblastoma cells. Taken together, EpCAM conjugated MSN might be advantageous for the targeted treatment of receptor-overexpressed ocular malignancies like retinoblastoma.

\section{Acknowledgement}

The study was funded by Natural Science Foundation of Heilongjiang Province of China (H201394) and Doctoral Science Foundation of the 2nd Affiliated Hospital of Haerbin Medical University.

\section{References}

1. B.M. Sanders, G.J. Draper, J.E. Kingston, Retinoblastoma in Great Britain 1969-80: incidence, treatment, and survival, Br. J. Ophthalmol. 72 (1988) 576-58.

2. J.A. Shields, C.L. Shields, Current Management of Retinoblastoma, Mayo Clin. Proc. 69 (1994) 50-56.

3. C.G. Rodriguez, M.W. Wilson, G. Chantada, L. Fu, I. Qaddoumi, C. Antoneli, C. Leal-Leal, et al., Retinoblastoma: one world, one vision, Pediatrics 122 (2008) e763e770.

4. K.R. Van Quill, P.K. Dioguardi, C.T. Tong, J.A. Gilbert, T.M. Aaberg Jr., H.E. Grossniklaus, et al., Subconjunctival carboplatin in fibrin sealant in the treatment of transgenic murine retinoblastoma, Ophthalmology 112 (2005) 1151-1158 
5. F. Doz, F. Khelfaoui, V. Mosseri, et al., The role of chemotherapy in orbital involvement of retinoblastoma, The experience of a single institution with 33 patients, Cancer 74 (1994) 722-732.

6. D.H. Abramson, C.M. Frank, I.J. Dunkel, A phase I/II study of subconjunctival carboplatin for intraocular retinoblastoma, Ophthalmology 106 (1999) 1947-1950

7. H.S. Chan, M.D. Canton, B.L. Gallie, Chemosensitivity and multidrug resistance to antineoplastic drugs in retinoblastoma cell lines, Anticancer Res. 9 (1989) 469-474

8. B.P. Marr, I.J. Dunkel, A. Linker, D.H. Abramson, Periocular carboplatin for retinoblastoma: long-term report (12 years) on efficacy and toxicity, Br. J. Ophthalmol 96 (2012) 881-883.

9. F.P. Manjandavida, S.G. Honavar, V.A.P. Reddy, R. Khanna, Management and outcome of retinoblastoma with vitreous seeds, Ophthalmology 121 (2014) 517-524.

10. D. Shome, S.G. Honavar, V.A. Reddy, The role of periocular carboplatin as an adjunctive therapy in advanced intraocular retinoblastoma, Proceedings of the Annual Meeting of the American Academy of Ophthalmology; October; 15-18; Chicago, USA, 2005

11. I. Schmack, G.B. Hubbard, S.J. Kang, T.M. Aaberg Jr, H.E. Grossniklaus, Ischemic necrosis and atrophy of the optic nerve after periocular carboplatin injection for intraocular retinoblastoma, Am. J. Ophthalmol 142 (2006) 310-315.

12. H. Kiratli, S. Kocabeyoglu, S. Bilgic, Severe pseudo-preseptal cellulitis following subTenon's carboplatin injection for intraocular retinoblastoma, J. AAPOS 11 (2007) 404405. 
13. T. Ramasamy, J.H. Kim, J.Y. Choi, T.H. Tran, H.G. Choi, C.S. Yong, J.O. Kim, pH sensitive polyelectrolyte complex micelles for highly effective combination chemotherapy, J. Mat. Chem. B 2 (2014) 6324.

14. T.H. Tran, T. Ramasamy, D.H. Troung, H.G. Choi, C.S. Yong, J.O. Kim. Preparation and characterization of fenofibrate-loaded nanostructured lipid carriers for oral bioavailability enhancement, AAPS PharmSciTech. 15 (2014) 1509.

15. P. Yang, S. Gai, J. Lin, Functionalized mesoporous silica materials for controlled drug delivery, Chem Soc Rev. 41 (2012) 3679-98.

16. C. Argyo, V. Weiss, C. Bra"uchle, B. Thomas, Multifunctional Mesoporous Silica Nanoparticles as a Universal Platform for Drug Delivery, Chem Mate. 26(2013) 435-51.

17. M. Mitra, M. Kandalam, R.S. Verma, K. UmaMaheswari, S. Krishnakumar, Genome wide changes accompanying knock-down of Ep-CAM in retinoblastoma, Mol. Vis 16 (2010) 828-42.

18. W.A. Osta, Y. Chen, K. Mikhitarian, M. Mitas, M. Salem, Y.A. Hannun, D.J. Cole, W.E. Gillanders, Ep-CAM is overexpressed in breast cancer and is a potential target for breast cancer gene therapy, Cancer Res. 64 (2004) 5818-24.

19. M.J. Winter, I.D. Nagtegaal, J.H. van Krieken, S.V. Litvinov, The epithelial cell adhesion molecule (Ep-CAM) as a morphoregulatory molecule is a tool in surgical pathology, Am. J. Pathol 163 (2003) 2139-48.

20. H. Koprowski, Z. Steplewski, K. Mitchell, M. Herlyn, D. Herlyn, P. Fuhrer, Colorectal carcinoma antigens detected by hybridoma antibodies, Somatic Cell Genet. 5 (1979) 95771. 
21. A. del Pozo-Rodríguez, D. Delgado, M.A. Solinís, A.R. Gascón, J.L. Pedraz, Int. J.Pharm. 360 (2008) 177-183.

22. Y.M.M.E. Barnett, D. Takemoto, H. Davidson, U.B. Kompella, Mol. Vis. 23 (2007) $746-757$

23. C.L. Shields, P. De Potter, B.P. Himelstein, J.A. Shields, A.T. Meadows, J.M. Maris, Arch. Ophthalmol. 114 (1996) 1330-1338.

24. F. Griesinger, M. Metz, L. Trumper, T. Schulz, D. Haase, Lung Cancer 44 (2004) $261-265$.

25. L.S. Jabr-Milane, L.E. van Vlerken, S. Yadav, M.M. Amiji, Cancer Treat Rev. 34 (2008) 592-602. 


\section{Figure captions}

Figure 1: Schematic presentation of preparation of EpCMSN nanocarrier system

Figure 2: (a) Particle size distribution of EpCMSN; (b) TEM imaging of EpCMSN; (c) gel electrophoresis of EpCAM conjugation.

Figure 3: In vitro drug release of CRB from EpCMSN. The release study was performed by dialysis method and analyzed by HPLC method.

Figure 4: Comparative cellular uptake of CMSN and EpCMSN in retinoblastoma cancer cells. The cellular uptake was studied by means of flow cytometer

Figure 5: Subcellular distribution of EpCMSN in Y79 cancer cells. Rhodamine-B was used as a fluorescent marker to track the distributions.

Figure 6: In vitro cell viability assay of free CRB, CMSN, and EpCMSN in Y79 cancer cells. The cell viability was determined by MTT assay.

Figure 7: Caspase-3 assay of free CRB, CMSN, and EpCMSN in Y79 cancer cells.

Figure 8: Annexin V and PI based apoptosis assay. The Y79 cancer cell was treated with respective formulations and the apoptosis was checked by flow cytometer. 

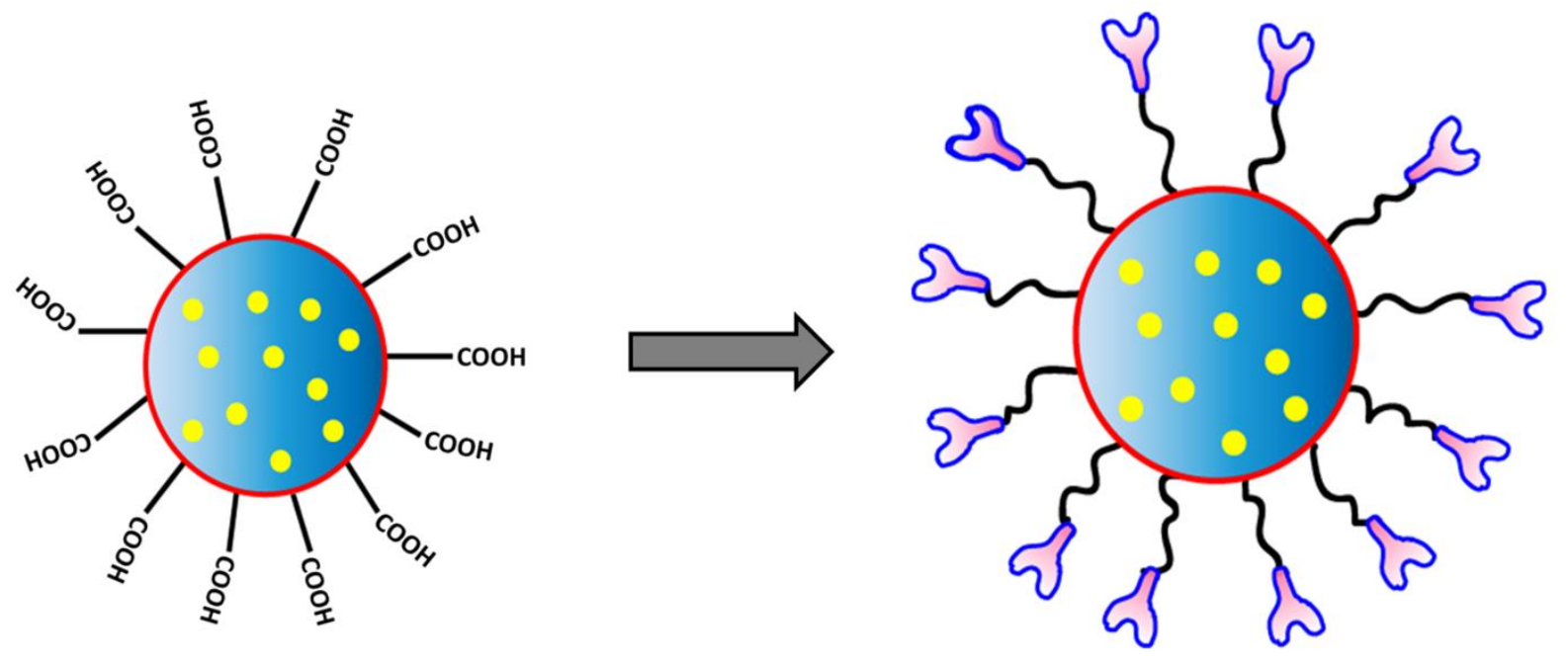

Carboplatin-loaded MSN

EpCAM/Carboplatin-loaded MSN

$\bigcirc \mathrm{nCOOH}+\varepsilon_{\mathrm{NH2}}^{\stackrel{\mathrm{EDC} / \mathrm{NHS}}{\longrightarrow}} \bigcirc \mathrm{uCO}-\mathrm{NH} \backsim$

Figure 1 
(a)

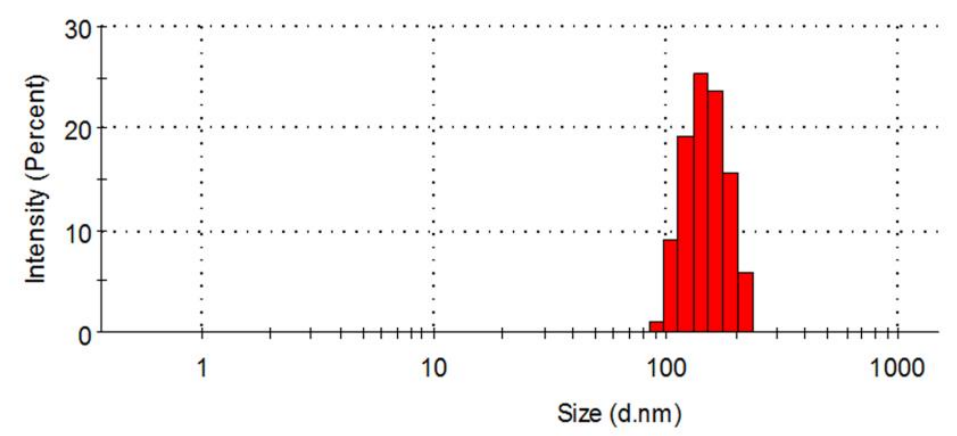

(b)

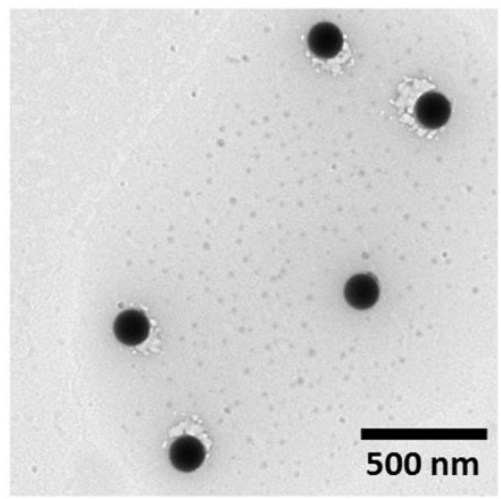

$100 \%$

conjugation

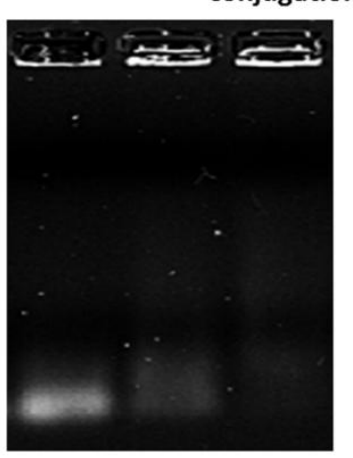

(c)

Figure 2 


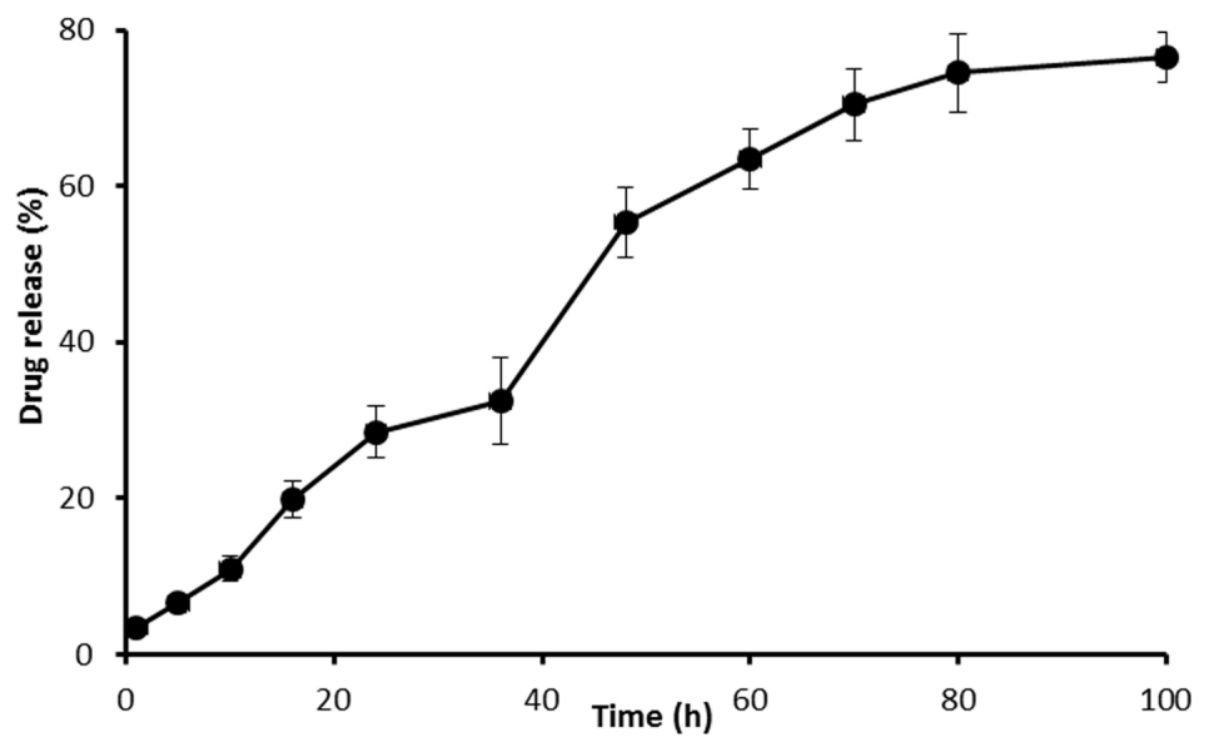

Figure 3 


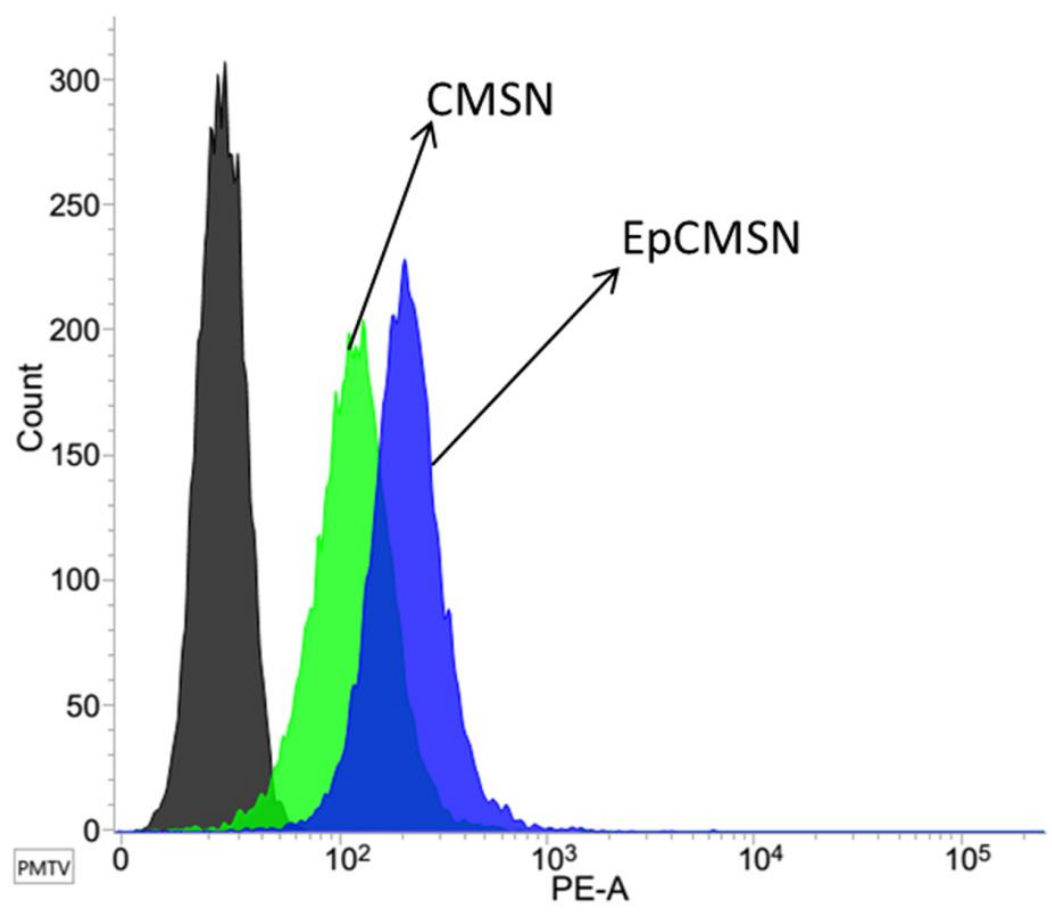

Figure 4 

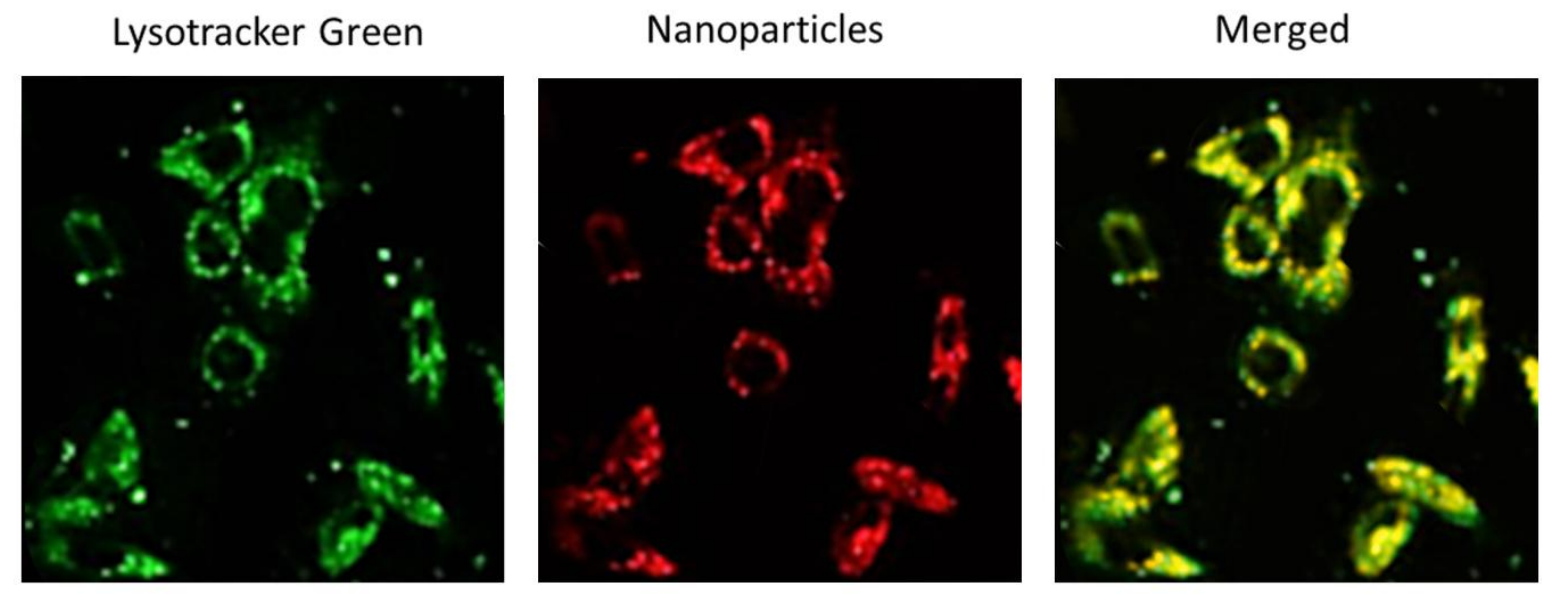

Figure 5 


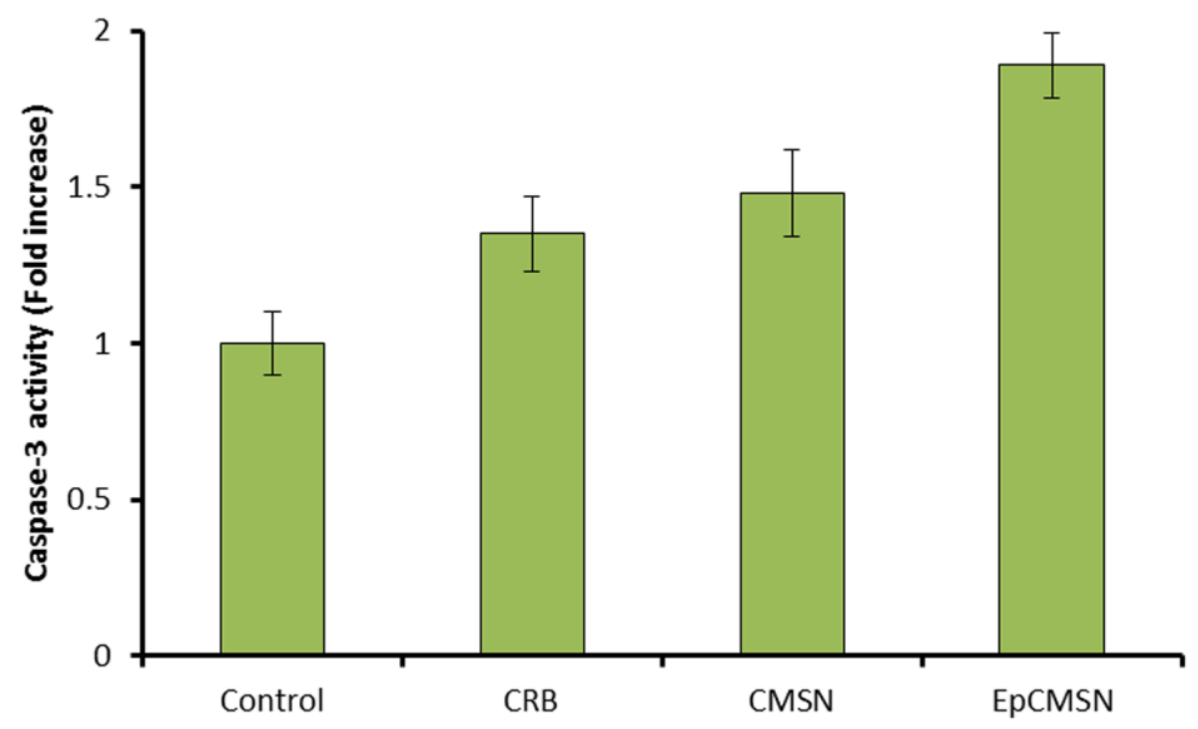

Figure 6 


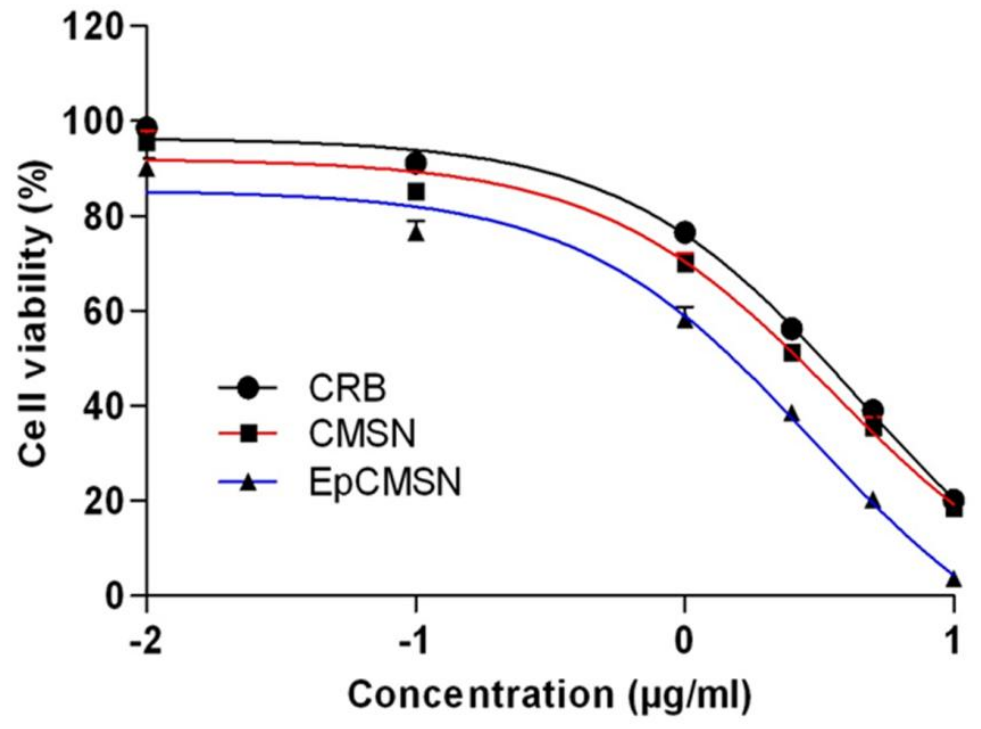

Figure 7 

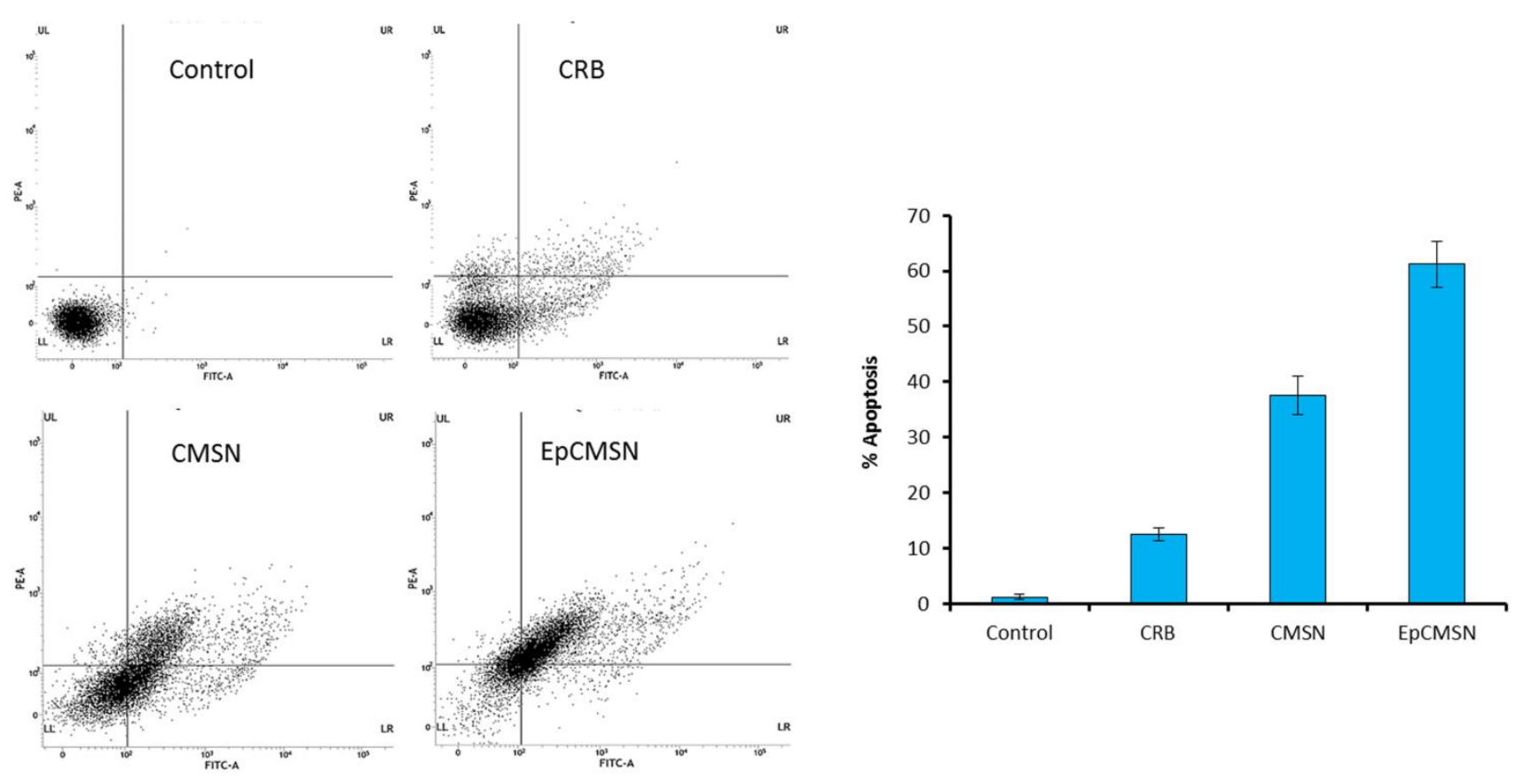

Figure 8 